\title{
Feedback literacy
}

David Carless, David Boud

\section{Source}

David Carless, David Boud. (2018). The development of student feedback literacy: enabling uptake of feedback. Assessment \& Evaluation in Higher Education, vol. 43 (8), 1315-1325. doi:10.1080/02602938.2018.1463354.

'The perceptions, capabilities and characteristics needed to make sense of information and use it to enhance work or learning strategies' 